\title{
Design and Implementation of Office Automation System based on Web Service Framework and Data Mining Techniques
}

\author{
He Huang ${ }^{1, a}$ \\ ${ }^{1}$ Sichuan Vocational and Technical College, Suining City, Sichuan Province. \\ axvnzph@163.com
}

Keywords: Office Automation, Web Service, Data Mining, Systematic Implementation.

\begin{abstract}
Smart mobile devices, the application of the social network media and the information behavior main body of increasingly frequent activity, has produced in these types of equipment, media and behavior diversity, complex structure, a large number of unstructured data and semi-structured data quantity has increased dramatically, human data into a driving force for social development is one of the era of big data. Under this background, we conduct research on office automation system based on web service framework and data mining techniques. We design and implement the system with the assistance of machine learning algorithms as well as the web service framework and achieve the satisfactory result which serves as the foundation for our future research.
\end{abstract}

\section{Introduction}

Hereinafter referred to as OA office automation, is the use of network communication foundation and advanced network application platform, the construction of safe, reliable, efficient, open electronic office automation and information management system, provide enterprises with modern daily office conditions and the abundant comprehensive information service, to realize the automatic document management and office automation transaction, to improve office efficiency and management level, realize the standardization of the enterprise each department daily business work, electronic, and the standardization. Modern enterprise management needs to rapid transmission and processing of large amounts of information, the traditional enterprise information communication methods cannot meet this need, so should be potential and office automation management system, and developed rapidly. It can meet the needs of enterprise office efficiency, automation and information technology effectively improve the whole management level of enterprises, to promote enterprise's influence on society that greatly enhance the core competitiveness of the enterprise [1].

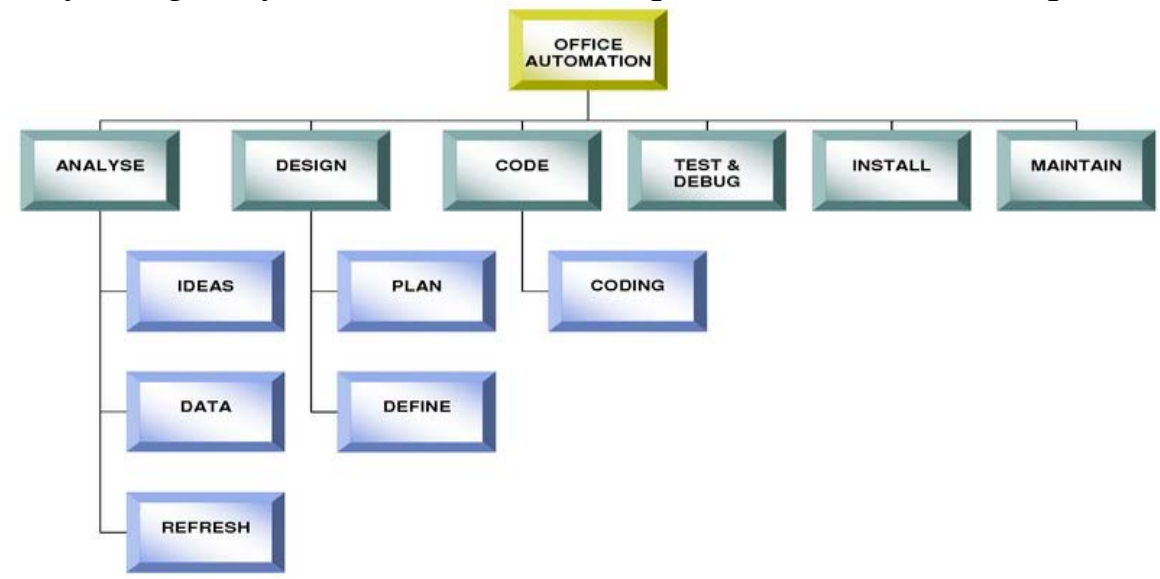

Fig. 1 The Primary Components of the Office Automation Framework

As illustrated in the figure one, the primary components of the OA framework is demonstrated. Traditional information management in software development is a blend of form basic styles, process jump control, database access to information, such as operation, so even if the customer need to form or to make small changes to the process, the upper software need to be modified, the corresponding requirement and also to the underlying database in basic modification operations, to meet customer 
requirements. The change of the whole system and the maintenance workload is big which is not conducive to the implementation of the whole system. After the introduction of workflow technology, system can better adapt to the enterprise business process reengineering with better documentation, information or tasks according to the rules set by the group between participants free delivery, its core is to make the business process dynamically configurable programming, which greatly increased the flexibility of OA software and can effectively solve the lonely phenomenon and caused the enough understanding of the connotation of the OA problem [2].

Under this background, in this paper, we conduct theoretical analysis on the implementation of the office automation system based on web service framework and data mining techniques. The rest of the paper is organized as follows. (1) We review and introduce concepts and systematic architecture of web service framework to serve as the basis. (2) We integrate the data mining technique to enhance the effectiveness and robustness of the traditional OA system. (3) We implement the proposed system with the detailed discussion of the inner logistics. (4) We summarize the work with final conclusion.

\section{Implementation of the Proposed OA System}

The Web Service Framework. Web service is a new kind of the Web application. It through the lightweight and has nothing to do with the suppliers' communication protocol to accept and handle from other systems on the network of the request of the XML format for design.

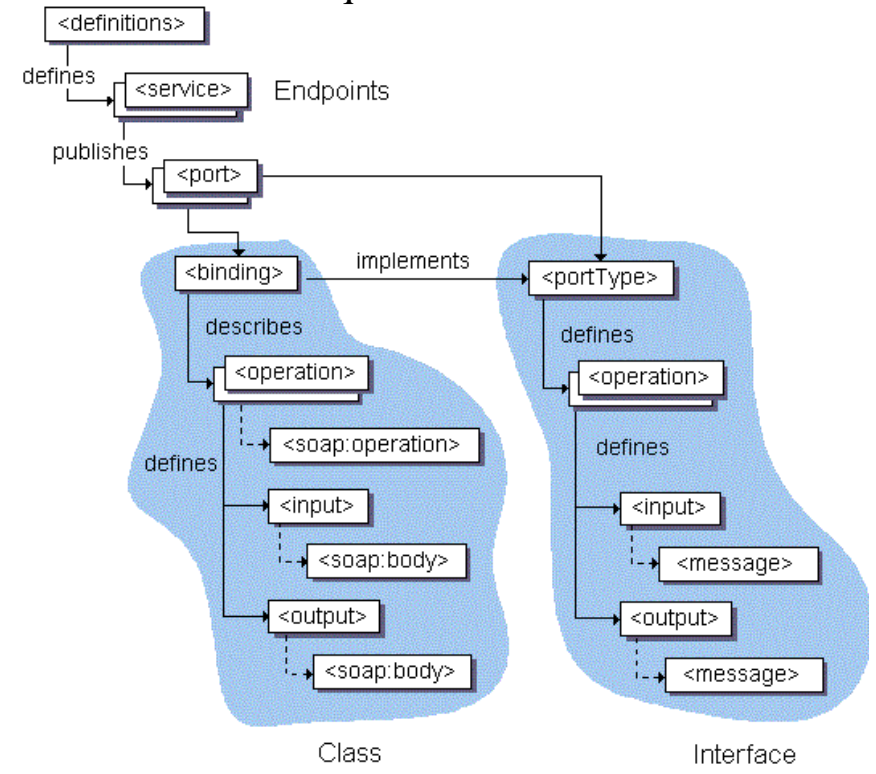

Fig. 2 The Sample Architecture of the Web Service Framework

Web service architecture shows elements instantiation and realization way of operation, different roles with different artifacts for different operation to implement the web service. In general, the core components of the system could be separated into the listed aspects. (1) Service provider: it refers to the owner of the service to other services and provides the function of the existing, from the point of view of system structure, it refers to provide access service platform. (2) Service broker: it refers to the database used to store information service description, service providers and service requester lookup service and obtain the service binding information and it serves as the role of the manager. (3) Service requesters: it refers to the enterprise or individual to a specific service, users of the service function, from the point of view of the system structure, it refers to find and invoke the service client application. Simple object access protocol SOAP is a based on the XML has nothing to do with the platform system communication protocol is a kind of message specification, it describes the data type of the message format, and a set of serialization rules, including structured types and arrays [3].

When the flow distribution in the enterprise internal workflow on the server, other main business processes for process called directly through the distributed component layer. Advocate tone server event trigger to call remote sub-processes event, the event handler according to receive events and scenarios for appropriate treatment and was moved to the server to create process instances request 
message and establish a session. Session content mainly includes the main process can logo, internal process node related data with remote process the data and the caller.

Advocate tone server receives successfully create the instance message, was moved to the server Settings related data request message. Modulated server parsed out relevant data in the message, set to the corresponding process, and returns the response message. If it is synchronous interaction advocate tone server waiting to be modulated server operation to complete and return the results and related data information, advocate the parse the message and set the relevant data to the process.

Data Mining Technique. Data mining as an important direction of artificial intelligence, because of the widely used, developing very rapidly, data mining is extracted from a large amount of data a credible, novel and effective and can be understood model of advanced treatment process. The task of the data mining from the data found in the model. Pattern has a lot of kinds, according to the feature points are two broad categories: prediction model and the model of description. Prediction model can be based on the value of the data item accurately determine the pattern of a certain result. Descriptive model is a description of the rules of data exists in the, or according to the similarity of data grouping the data. As the basis, Bayesian theory shown in the formula one undertakes the vital role [4].

$$
P(c \mid x)=P(x \mid c) \cdot P(c) / P(x)
$$

According to Bayes' theorem to calculate the probability of a sample belongs to each category, and take the highest probability of class as the classification of the sample. Its advantage lies in the low computational cost as prediction and classification of similarity is to start by building a model, then using models to predict the unknown values. Correspondingly, we could separate the core data mining algorithms into the listed categories. (1) The neural network. Neural network is to simulate biological structure and function of the nervous system, is a kind of nonlinear prediction model through training to learn, can complete classification, clustering, characteristics of mining and other data mining tasks. The weights of neural network learning method are mainly manifested in revision. Its advantage is has anti-interference, nonlinear learning, associative memory function can get accurate prediction results for complex situation. (2) Fuzzy set method. Fuzzy set method using fuzzy set theory to problems in the fuzzy evaluation, fuzzy decision and the fuzzy pattern recognition and fuzzy clustering analysis. Membership to fuzzy set theory is used to describe properties of fuzzy things. (3) Genetic algorithm. Genetic algorithm is a genetic combination of crossover and mutation and natural selection, genetic operation to generate implementation rules, and machine learning method based on the theory of the evolution. (4) The rough set method. Rough set method also called, rough set theory is a new kind of dealing with vague, imprecise, incomplete mathematical tool as can process the data reduction, data correlation found, the meaning of data evaluation. Its advantage is simple algorithm as don't need any preparation about the data or additional letter and only use the standard of formula two.

$$
\operatorname{Standard}(i, j)=\sqrt{\left(\left|x_{i 1}-x_{j 1}\right|^{2}+\left|x_{i 2}-x_{j 2}\right|^{2}+\mathrm{L}+\left|x_{i p}-x_{j p}\right|^{2}\right)}
$$

Relatively, the mining procedures could be organized as follows. (1) Before data mining work, we want to clearly know the goal of data mining. Specified in mining business objectives, determine the evaluation method to achieve objectives. (2) Once established mining business goals, the next step is to select data to achieve the goal of this. These data may be a subset of the data warehouse or the data market that may be all the data in the online transaction processing system. (3) Once everywhere the large amounts of data collection, it needs to use the right tools to transform it into a suitable format. Whether audit data is to analyze the data structure is suitable for mining, and is suitable for mining tools. The quality of the data and the organization structure is the result of the knowledge of deadly effects, so before mining deal with existing data have a comprehensive and profound understanding, in order to smoothly handle a few steps behind. (4) Found conclusion and discuss business executives, the domain experts, to determine whether conclusion is correct and meet the demands of the business, and if the results of the mining errors will be looking for the wrong reasons to reconstruct model. 


\section{Systematic Architecture Verification}

In deep understanding of the open source project uEngine characteristics on the basis of combining the actual requirement of school's office, we design and realize the office system based on workflow technology. This system is by the workflow engine, process management tools, the process definition tool and ordinary user management of four parts, the various components of system, including the own data interface and forms that is shown in the figure three. In detail, our system can be separated into the listed aspects. (1) The workflow engine. uEngine workflow components named after open the kernel frame structure, OKF kernel need to manage all of the process control part, at the same time, when necessary, also need to activate control activities in the whole process instance life cycle. (2) The process manager. Process manager mainly includes the management and monitoring part, one part is the core of the process manager. Management section provides the perfect role management, system Settings, configuration file management and log management and the other functions. (3) The process definition. This part is on the basis of uEngine original designer for some of its controls on localization and modification, and increases the has the function of sending text messages and written agenda form more flexible with their characteristics of process design.

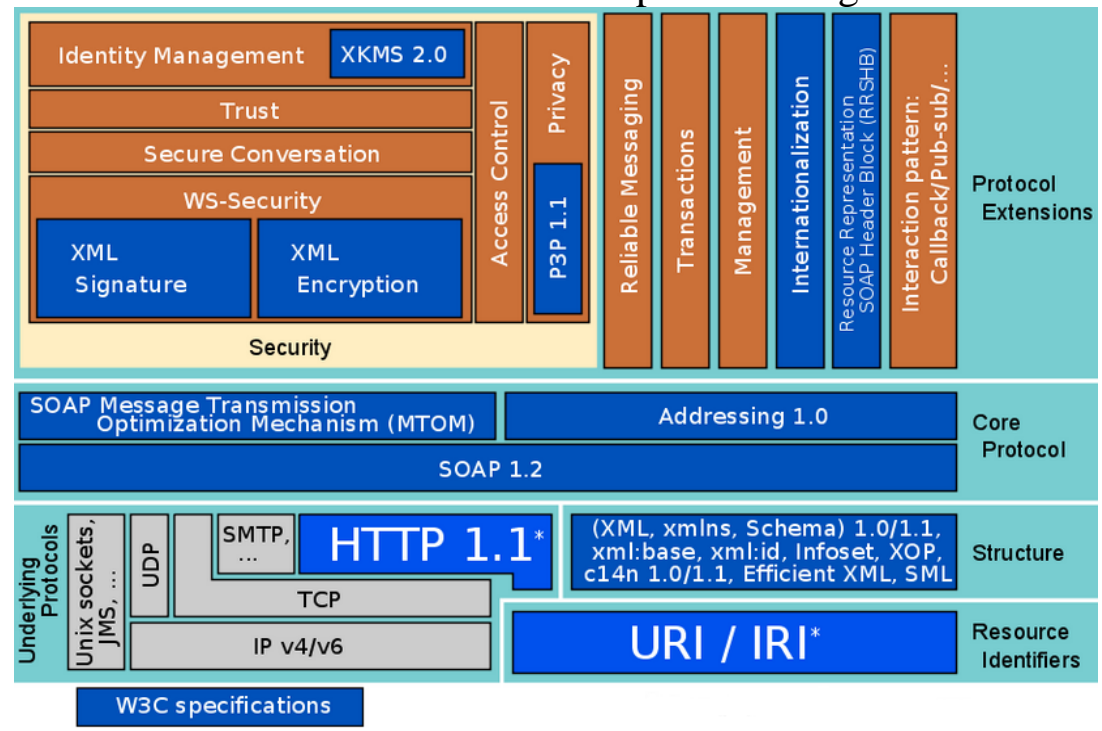

Fig. 3 The Systematic Architecture of the Proposed Office Automation System

\section{Summary and Conclusion}

In this paper, we design and implement the office automation system based on web service framework and data mining techniques. Office automation is the modern office and computer network function combination of a new type of office way, all in traditional office uses all sorts of new technology, new machines and equipment engaged in the business office, belongs to the field of office automation. We use form driven workflow engine to jump that can better realize the user free configuration process and form to better achieve the user requirements. Additionally, we combine the data mining technique to optimize the system architecture that will enhance the robustness.

\section{References}

[1] Wei, Zhao Yanzhi Yan. "Study on Implementation of Office Automation System Based on Company X." Office Informatization 2 (2012): 007.

[2] Yang, Yu Qiang, and Heng Chen. "Framework Structure on Enterprise Office Automation System." Applied Mechanics and Materials. Vol. 713. 2015.

[3] Jingying, Feng, and Li Shaohua. "Application of Reflective Teaching in Office Automation Course." Network Security Technology \& Application 9 (2014): 115. 
[4] Wang, An, and Xiang Qing Zhang. "System Design of E-Government Office Automation." Advanced Materials Research. Vol. 889. 2014. 